\title{
Interval Approach for Stability Analysis of a Pressurized Water Reactor with Parametric Uncertainty using LMI based H-infinity Controller
}

\author{
Shohan Banerjee, Jiamei Deng \\ School of Built Environment, Engineering and \\ Computing \\ Leeds Beckett University \\ United Kingdom, Leeds \\ s.banerjee@leedsbeckett.ac.uk \\ j.deng@leedsbeckett.ac.uk
}

\section{S.R. Shimjith}

Reactor Control System Design Section

Bhabha Atomic Research Centre, Homi Bhabha National

Mumbai, India

srshim@barc.gov.in

\author{
Vineet Vajpayee, Victor M.Becerra, Nils Bausch \\ School of Energy and Electronic Engineering \\ University of Portsmouth \\ United Kingdom, Portsmouth \\ vineet.vajpayee@port.ac.uk \\ victor.becerra@port.ac.uk \\ nils.bausch@port.ac.uk
}

\author{
John Arul \\ Reactor Shielding and Data Division \\ Indira Gandhi Centre for Atomic Research \\ Kalpakkam, India \\ arul@barc.gov.in
}

\begin{abstract}
The self-regulating and self-stabilizing natures of a Pressurized Water Reactor (PWR) make it most suitable for the nuclear power industry. However, handling the disturbance and stabilizing a PWR with the disturbance are highly challenging tasks for control engineers due to inherent nonlinearity in a reactor. It leads to change in the behavior with variation in reactor power. Further, plenty of uncertainties exist in a PWR due to the heat transfer from fuel to coolant and the reactivity changes due to component faults and variation in fuel and coolant temperatures. Thus, it is essential to design a robust controller which can stabilize the system in presence of uncertainties and disturbances as well. In this paper, a state space model has been considered using the point kinetics equations of PWR coupled with the Mann's thermal-hydraulic equations. The system matrices have been evaluated at different power levels with uncertainty in parameters to produce an interval state space model. A $\boldsymbol{H}_{\infty}$ based Full State Feedback Controller (SFSC) has been designed for this interval plant and then used for establishing a stability criterion in presence of disturbances. The outcomes have been validated using MATLAB simulations and discreetly exemplified in the result section.
\end{abstract}

Index Terms- H-infinity controller, Interval system, PWR, State feedback controller.

\section{INTRODUCTION}

In nuclear power plants (NPPs), pressurized water reactors (PWRs) are popular due to their self-regulating and self- stabilizing nature. However, control system design for PWRs is challenging due to inherent non-linear and time-varying nature of PWRs, which depends on the reactor thermal power [1]. Moreover, uncertainties in the measurements of actual reactor power and neutron flux add further problems and pose a challenge to design robust controllers for NPPs. Contemporary researchers have attempted to address the problem using various approaches such as, Full State Feedback with Integral plus Derivative (PID) controllers accompanied by Fractional Order (FO) phase shapers and Fractional Order PID (FOPID) controllers [5]. Optimal Full State Feedback Controllers (FSFCs) are known to procure Controllers (FSFC) [2], robust Fuzzy Logic Controllers (FLCs) [3], Periodic Output Feedback Techniques [4] to Proportional robust designs [6] although they do not necessarily guarantee a desired time response under bounded parametric uncertainties. FLC are well-known for their uncertainty handling capacities. Some researchers [3] have also proposed the use of FLCs for control of uncertain system, but such controllers are difficult to validate from a stability point of view.

Controller design for PWR remains a challenging task, especially during power maneuvering. Researchers have attempted to address the problem of control design during load-following operation of a PWR. Out of the various existing techniques, Model Predictive Control (MPC) has been studied extensively. Kim et al. [7] used MPC to design a controller for controlling the power distribution and level. They used a genetic algorithm to optimize the discrete control rod speed. In [8], Eliasi et al. developed robust MPC by 
imposing constraints on states during optimization. State estimators based MPC design has been considered in [9]. Apart from MPC, other control design techniques have been used during load-following. Dong et al. [10] used feedback dissipating controller with nonlinear observer having a Hamiltonian structure for a Single Input Single Output (SISO) system and extended it to design a controller for a low temperature PWR. Torabi et al. [11] proposed a controller design for power regulation of a PWR using Quantitative Feedback Theory (QFT) approach. In [12], adaptive controller design methodology was adopted for the power level control of a PWR, where the designed controller guaranteed global asymptotic stability. Bose et al. [13,14] designed a load following operation for small PWR using nonlinear dynamic inversion (NDI) technique showing good tracking capabilities. However, the approach cannot guarantee stability of the system.

It is a well-known fact that in industry, fixed order or structured controllers are preferred for output feedback problems, owing to their simple usage. In real scenario, $90 \%$ of the structured controllers are traditional PID controllers due to disturbance robustness, good performance and easy implementation [15]. However, these types of controllers do not always guarantee closed-loop system stability and do not ensure performance constraints, especially for NPPs. Errorless temperature measurement of coolant and estimation of fuel temperature are challenging tasks. Therefore, there is a possibility to shift closed-loop Eigen values due to the improper measurement of fuel and coolant temperature.

However, it is difficult to design the demanded structure to overcome some basic performance requirements. The most common performance criteria are minimizing the $H_{\infty}$ norms of the transfer function between performance output and disturbance input, and some necessary parameters, such as maximum overshoot and settling time, which is related to the dynamic response of the system.

In recent work, Yan et al in [16] proposed an interesting robust power controller for a small, pressurized water reactor based on $H_{\infty}$ and mixed sensitivity method. In this method, a multi-input and multi-output transfer function is developed. After that, five local controllers were designed for five power ranges, a multi-model approach with triangular membership functions was employed to integrate the five local controllers into a multi-model robust control system. Thus, the controller can work for the entire power range. However, in this paper, researchers did not provide information on how much disturbance tolerance could be handled.

In this paper, the parametric uncertainties due to thermal modeling are tackled by considering a spread of the reactor parameters within an interval, and the resultant model is then used to design a Linear Matrix Inequality (LMI) based $H_{\infty}$ FSFC, for a PWR. The proposed methodology is capable of handling uncertainties over an interval period and allows the plant to be controlled by a single optimal FSFC.

The rest of the paper is organized as follows. Section II describes the interval state space model of a PWR. Section III proposes the $H_{\infty}$ based FSFC controller design using the LMI and establishes a stability criterion for the uncertainties. Section IV shows the corresponding simulation result for a
PWR in presence of disturbances. Section IV concludes the paper.

\section{PWR WITH THERMAL HYDRAULIC MODEL}

In this section, an interval state space model is obtained for a PWR. A normalized point kinetic model of a PWR has been considered with a thermal hydraulic model. The Xenon and Iodine dynamics have little effects during total power control, so they are not considered here.

The dynamic model is given by:

$$
\begin{aligned}
& \frac{d P_{n}}{d t}=\frac{\rho_{t}-\sum_{i=1}^{6} \beta_{i}}{\Lambda} P_{n}+\sum_{i=1}^{6} \frac{\beta_{i}}{\Lambda} C_{i n} \\
& \frac{d C_{i n}}{d t}=\lambda_{i} P_{n}-\lambda_{i} C_{i n}, \quad i=1,2 \ldots 6 .
\end{aligned}
$$

where $P_{n}$ is neutronic power, $\rho_{t}$ is total reactivity, $\Lambda$ is neutron generation time, $\lambda_{i}, \beta_{i}$, and $C_{i n}$ are decay constant, fraction of delayed neutrons, and delayed neutron precursors' concentration of $i^{\text {th }}$ group, respectively.

The core thermal-hydraulics model is given by Mann's model [17] which considers two coolant lumps for every fuel lump,

$$
\begin{gathered}
\frac{d T_{f}}{d t}=H_{f} P_{n}-\frac{1}{\tau_{f}}\left(T_{f}-T_{c 1}\right), \\
\frac{d T_{c 1}}{d t}=H_{c} P_{n}+\frac{1}{\tau_{c}}\left(T_{f}-T_{c 1}\right)-\frac{2}{\tau_{r}}\left(T_{c 1}-T_{c i n}\right), \\
\frac{d T_{c 2}}{d t}=H_{c} P_{n}+\frac{1}{\tau_{c}}\left(T_{f}-T_{c 1}\right)-\frac{2}{\tau_{r}}\left(T_{c 2}-T_{c 1}\right) .
\end{gathered}
$$

where $T_{f}$ is average fuel temperature; $T_{c 1}$ and $T_{c 2}$ are average coolant temperatures in node 1 and node 2 , respectively; $T_{\text {cin }}$ is inlet temperatures of the first coolant node; $H_{f}$ and $H_{c}$ characterize the rate of rise of fuel and coolant temperatures respectively; $\tau_{f}$ and $\tau_{c}$ are time constants representing mean time for heat transfer from fuel to coolant and from core outlet to inlet, respectively while $\tau_{r}$ represents coolant residence time in the core. The heat transfer coefficient from fuel to coolant is assumed to be constant.

In this paper, only the power control loop without secondary side coolant heat transfer has been considered. The change in total reactivity is considered due to the control rod movement and reactivity feedback due to fuel and coolant temperature change. Here, a control rod acts as an actuator and this actuator movement can be represented by following equation:

$$
\frac{d \rho_{e x}}{d t}=G z_{c}
$$


where $\rho_{e x}$ is the external reactivity injected to the reactor core due to the control rod movement, $G$ is the reactivity worth of control rod while $z_{c}$ is the speed of the control rod movement.

The total reactivity can be obtained by

$\rho_{t}=\rho_{e x}+\alpha_{f} T_{f}+\alpha_{c} T_{c 1}+\alpha_{c} T_{c 2}$

where, $\alpha_{f}$ and $\alpha_{c}$ represent the temperature coefficients of the reactivity due to fuel and coolant, respectively.

Equations (1) to (7) are used to develop a linearized statevector model for the PWR form of:

$\dot{\boldsymbol{x}}(t)=\boldsymbol{A} \boldsymbol{x}(t)+\boldsymbol{B} \boldsymbol{u}(t)$ and $y(t)=\boldsymbol{C} \boldsymbol{x}(t)$

where

$\boldsymbol{x}=\left[\begin{array}{lllllllllll}\rho_{e x} & P & C_{1} & C_{2} & C_{3} & C_{4} & C_{5} & C_{6} & T_{f} & T_{c 1} & T_{c 2}\end{array}\right]^{T}$ denotes the set of state variables around an equilibrium state, input $\boldsymbol{u}$ is considered as control rod speed and output matrix $\boldsymbol{C}$ has been considered only with the reactor power of the system. $\boldsymbol{A}, \boldsymbol{B}$ and $\boldsymbol{C}$ can be computed by (9)-(11).

$\boldsymbol{A}=\left[\begin{array}{ccccccccccc}0 & 0 & 0 & 0 & 0 & 0 & 0 & 0 & 0 & 0 & 0 \\ 0 & -\frac{\beta}{\Lambda} & \lambda_{1} & \lambda_{2} & \lambda_{3} & \lambda_{4} & \lambda_{5} & \lambda_{6} & \frac{P_{0} \alpha_{f}}{\Lambda} & \frac{P_{0} \alpha_{c}}{\Lambda} & \frac{P_{0} \alpha_{c}}{\Lambda} \\ 0 & \frac{\beta_{1}}{\Lambda} & -\lambda_{1} & 0 & 0 & 0 & 0 & 0 & 0 & 0 & 0 \\ 0 & \frac{\beta_{2}}{\Lambda} & 0 & -\lambda_{2} & 0 & 0 & 0 & 0 & 0 & 0 & 0 \\ 0 & \frac{\beta_{3}}{\Lambda} & 0 & 0 & -\lambda_{3} & 0 & 0 & 0 & 0 & 0 & 0 \\ 0 & \frac{\beta_{4}}{\Lambda} & 0 & 0 & 0 & -\lambda_{4} & 0 & 0 & 0 & 0 & 0 \\ 0 & \frac{\beta_{5}}{\Lambda} & 0 & 0 & 0 & 0 & -\lambda_{5} & 0 & 0 & 0 & 0 \\ 0 & \frac{\beta_{6}}{\Lambda} & 0 & 0 & 0 & 0 & 0 & -\lambda_{6} & 0 & 0 & 0 \\ 0 & H_{f} & 0 & 0 & 0 & 0 & 0 & 0 & -\frac{1}{\tau_{f}} & \frac{1}{\tau_{f}} & 0 \\ 0 & 0 & 0 & 0 & 0 & 0 & 0 & 0 & \frac{1}{\tau_{c}} & -\left(\frac{1}{\tau_{c}}+\frac{2}{\tau_{r}}\right) & 0 \\ 0 & 0 & 0 & 0 & 0 & 0 & 0 & 0 & \frac{1}{\tau_{c}} & -\left(\frac{1}{\tau_{c}}-\frac{2}{\tau_{r}}\right) & -\frac{2}{\tau_{r}}\end{array}\right](9)$

$\boldsymbol{B}=\left[\begin{array}{lllllllllll}G & 0 & 0 & 0 & 0 & 0 & 0 & 0 & 0 & 0 & 0\end{array}\right]^{T}$

$\boldsymbol{C}=\left[\begin{array}{lllllllllll}0 & 1 & 0 & 0 & 0 & 0 & 0 & 0 & 0 & 0 & 0\end{array}\right]$

Next, $\boldsymbol{A}$ and $\boldsymbol{B}$ matrices have been evaluated using parameter values at different power level and hence an interval matrix can be represented as follows:

$\left.\boldsymbol{A}_{i} \in\left[\boldsymbol{A}_{60}, \boldsymbol{A}_{100}\right]\right)$

$\left.\boldsymbol{B}_{i} \in\left[\boldsymbol{B}_{60}, \boldsymbol{B}_{100}\right]\right\}$

where, $\boldsymbol{A}_{60}$ and $\boldsymbol{B}_{60}$ are the infimum, $\boldsymbol{A}_{100}$ and $\boldsymbol{B}_{100}$ are the supremum of the corresponding $\boldsymbol{A}_{i}$ and $\boldsymbol{B}_{i}$ matrices. Further an uncertainty has been injected into the thermal hydraulic parameter at the corresponding power level and the range of the interval has been increased and defined by:

$\left.\boldsymbol{A}_{i} \in[\underline{\boldsymbol{A}}, \overline{\boldsymbol{A}}]\right)$

$\left.\boldsymbol{B}_{i} \in[\underline{B}, \bar{B}]\right\}$

where, $\underline{\boldsymbol{A}}$ and $\boldsymbol{B}$ are the infimum, $\overline{\boldsymbol{A}}$ and $\overline{\boldsymbol{B}}$ are the supremum of the corresponding $\boldsymbol{A}_{i}$ and $\boldsymbol{B}_{i}$ matrices at power level 60 and 100 respectively and this notation has been used rest of this paper. These interval system matrices also used for the design of the H-infinity controller as well as to establish and analysis the stability criteria for the uncertain interval system.

\section{III. $H_{\infty}$ CONTROLler Design USING LMI}

For designing an LMI based $H_{\infty}$ controller for the system (13) a generalised schematic model has been considered, shown in Fig. 1.

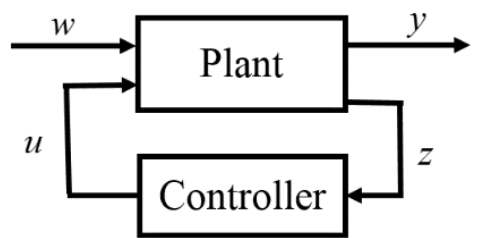

Fig. 1. Schematic diagram of generalised system.

To obtain the controller gain, the uncertain system can be represented by following state space equations [18]:

$$
\left.\begin{array}{l}
\dot{\boldsymbol{x}}=\boldsymbol{A} \boldsymbol{x}+\boldsymbol{B}_{1} u+\boldsymbol{B}_{2} w \\
y=\boldsymbol{C}_{1} \boldsymbol{x}+\boldsymbol{D}_{11} u+\boldsymbol{D}_{12} w \\
z=\boldsymbol{C}_{2} \boldsymbol{x}+\boldsymbol{D}_{21} u+\boldsymbol{D}_{22} w
\end{array}\right\}
$$

where, $w$ is the external disturbance signal, $u$ is the input signal, $y$ is the output signal and $z$ is the measured output signal.

For this PWR system, as stated in an earlier section, only the power loop of the PWR has been considered for the simulation and the secondary side heat transfer has not been considered. When only the power control loop is considered, there are two inputs, first the control rod speed, $\mathrm{z}$ and the second is the inlet temperature of the primary coolant, $T_{\text {Cin }}$. For simplicity, in power loop the $T_{\text {Cin }}$ may be taken as a constant and hence the only controllable input is control rod speed, $z_{c} . B_{l}$ is similar to (10). However, in a real-world scenario $T_{C i n}$ is not constant and mostly depends on the secondary coolant side. So, in this case $T_{C i n}$ has been considered as an input $B_{2}$, i.e., an input to the system, which is not controllable for the power loop and is described as follows:

$\boldsymbol{B}_{2}=\left[\begin{array}{lllllllllll}0 & 0 & 0 & 0 & 0 & 0 & 0 & 0 & 0 & T_{c i n} & 0\end{array}\right]$

Similarly, $C_{1}$ is the same with the output matrix i.e. (11) and $C_{2}$ is the full state identity matrix can be defined as

$\boldsymbol{C}_{2}=\left[\boldsymbol{I}_{11 \times 11}\right]$

And all the disturbance inputs $\left(D_{11} D_{12} D_{21} D_{22}\right)$ are initially kept as zero.

Further, the plant can be expressed as:

$p(s)=\left[\begin{array}{ccc}\boldsymbol{A} & \boldsymbol{B}_{1} & \boldsymbol{B}_{2} \\ \boldsymbol{C}_{1} & \boldsymbol{D}_{11} & \boldsymbol{D}_{12} \\ \boldsymbol{C}_{2} & \boldsymbol{D}_{21} & \boldsymbol{D}_{22}\end{array}\right]=\left[\begin{array}{cc}\boldsymbol{A} & \boldsymbol{B} \\ \boldsymbol{C} & \boldsymbol{D}\end{array}\right]$

The relationship of the input and output can be represented as:

$\left[\begin{array}{l}y \\ z\end{array}\right]=\left[\begin{array}{ll}p_{11} & p_{12} \\ p_{21} & p_{22}\end{array}\right]\left[\begin{array}{l}u \\ w\end{array}\right]$ 
And $u=\boldsymbol{K}_{f c} y$

By eliminating the measurement signal $z$ from (14) the closed loop transfer function from $u$ to $y$ can be represented as:

$T f_{u y}=f\left(p, \boldsymbol{K}_{f c}\right)=\left(p_{11}+p_{12} \boldsymbol{K}_{f c}\left(I-\boldsymbol{K}_{f c} p_{22}\right)^{-1} p_{21}\right)$

A standard $H_{\infty}$ control law can be established from the (20) which will ensure the closed loop system to be stable over a region and minimize the performance demand the norm of the transfer function matrix, can be represented as:

$J=\min \left\|T f_{u y}\right\|_{\infty}$

$\left.\Rightarrow \min \left\|\left(p_{11}+p_{12} \boldsymbol{K}_{f c}\left(I-\boldsymbol{K}_{f c} p_{22}\right)^{-1} p_{21}\right)\right\|_{\infty}=\gamma_{0}\right\}$

where, $\gamma_{0}$ is the minimum which is quite difficult to achieve. Thus, a suboptimal $H_{\infty}$ control has been chosen which gives a finite solution for the plant. In this case, the $H_{\infty}$ norm of the plant considered to be greater than $\gamma_{0}$ and can be represented as:

$J=\left\|T f_{u y}\right\|_{\infty}$

$\left.\Rightarrow\left\|\left(p_{11}+p_{12} \boldsymbol{K}_{f c}\left(I-\boldsymbol{K}_{f c} p_{22}\right)^{-1} p_{21}\right)\right\|_{\infty}<\gamma\right\}$

where, $\gamma \geq \gamma_{0}$, and this is also considered as the standard $H_{\infty}$ control problem.

\section{A. Controller Synthesis:}

Recently, many researchers have established $H_{\infty}$ control problem from the Riccati equation which can be easily solved by the LMI approach [18]. The main advantage of the LMI approach is that it can use the matrix operation to produce a direct controller gain for the system. If there is a positive definite matrix $\boldsymbol{P}=\boldsymbol{P}^{T}>0$ and a matrix $\boldsymbol{W}$ exists, a standard LMI equation for Riccati equation can be written as:

$\left[\begin{array}{ccc}\boldsymbol{A P}+\boldsymbol{B}_{2} \boldsymbol{W}+\left(\boldsymbol{A} \boldsymbol{P}+\boldsymbol{B}_{2} \boldsymbol{W}\right)^{T} & \boldsymbol{B}_{1} & \left(\boldsymbol{C}_{1} \boldsymbol{P}+\boldsymbol{D}_{12} \boldsymbol{W}\right)^{T} \\ \boldsymbol{B}_{1}^{T} & -\boldsymbol{I} & \boldsymbol{D}_{11}^{T} \\ \boldsymbol{C}_{1} \boldsymbol{P}+\boldsymbol{D}_{12} \boldsymbol{W} & \boldsymbol{D}_{11} & -\boldsymbol{I}\end{array}\right]<0$

From (23), the state feedback gain for system (14) can be expressed as:

$\boldsymbol{K}_{f_{c}}=\boldsymbol{W}(\boldsymbol{P})^{-1}$

Next, the suboptimal control performance for $H_{\infty}$ robust controller can be expressed as:

$$
\left.\begin{array}{l}
\left\|T f_{u y}\right\|_{\infty}<\gamma \\
\Rightarrow\left\|\gamma^{-1} T f_{u y}\right\|_{\infty}<1
\end{array}\right\}
$$

The output matrix and output disturbance matrices can be represented as $\boldsymbol{C}_{1} \gamma^{-1}, \boldsymbol{D}_{11} \gamma^{-1}$ and $\boldsymbol{D}_{12} \gamma^{-1}$, respectively, which are used in (23) to create a new LMI as:

$\left[\begin{array}{ccc}\boldsymbol{A P}+\boldsymbol{B}_{2} \boldsymbol{W}+\left(\boldsymbol{A P}+\boldsymbol{B}_{2} \boldsymbol{W}\right)^{T} & \boldsymbol{B}_{1} & \gamma^{-1}\left(\boldsymbol{C}_{1} \boldsymbol{P}+\boldsymbol{D}_{12} \boldsymbol{W}\right)^{T} \\ \boldsymbol{B}_{1}^{T} & -\boldsymbol{I} & \boldsymbol{D}_{11}^{T} \\ \gamma^{-1}\left(\boldsymbol{C}_{1} \boldsymbol{P}+\boldsymbol{D}_{12} \boldsymbol{W}\right) & \gamma^{-1} \boldsymbol{D}_{11} & -\boldsymbol{I}\end{array}\right]<0(26)$
Now, if $\boldsymbol{A}$ and $\boldsymbol{B}_{1}$ are the interval matrices and if a single $\boldsymbol{P}$ exists over the interval then only the $\boldsymbol{K}_{f c}$ can handle the interval plant for the specified disturbance.

\section{B. Criteria for stability:}

If $\boldsymbol{A}$ is an interval matrix and $\boldsymbol{A} \in[\underline{\boldsymbol{A}}, \overline{\boldsymbol{A}}]$ then the Lyapunov stability function can be written as:

$\boldsymbol{A}^{T} \boldsymbol{P}+\boldsymbol{P} \boldsymbol{A}<0$

Considering $\boldsymbol{P A}=\boldsymbol{\psi}$, i.e. point matrix is multiplied by an interval matrix and generated a new interval matrix, $\boldsymbol{\psi} \in$ $[\underline{\psi}, \overline{\boldsymbol{\psi}}],[19]$ (interval matrix with point matrix multiplication)

$\left.\begin{array}{rl}\underline{\boldsymbol{\psi}} & =\boldsymbol{P} \boldsymbol{A}_{c}-|\boldsymbol{P}| \cdot \boldsymbol{A}_{r} \\ \overline{\boldsymbol{\psi}} & =\boldsymbol{P} \boldsymbol{A}_{c}+|\boldsymbol{P}| \cdot \boldsymbol{A}_{r}\end{array}\right\}$

Where, $\boldsymbol{A}_{c}$ and $\boldsymbol{A}_{r}$ are the nominal matrix and the uncertainty matrix of $\boldsymbol{A}$ which can be defined as:

$\left.\boldsymbol{A}_{c}=\frac{1}{2}(\underline{\boldsymbol{A}}+\overline{\boldsymbol{A}})\right\}$

$\left.\boldsymbol{A}_{r}=\boldsymbol{A}_{c}-\underline{\boldsymbol{A}}\right\}$

$\boldsymbol{A}^{T}$ is now the interval matrix and $\boldsymbol{P}$ is the point matrix, (interval matrix with point matrix multiplication) [19].

Let, $\mathbf{A}^{\mathrm{T}} \boldsymbol{\psi}=\chi \quad \mid \chi \in[\underline{\chi}, \bar{\chi}]$

$\underline{\chi}=\chi_{c}-\chi_{r}$

$\left.\bar{\chi}=\chi_{c}+\chi_{r}\right\}$

$\chi_{c}=\boldsymbol{A}_{c}^{T} \boldsymbol{P}$

$\left.\chi_{r}=\boldsymbol{A}_{r}^{T}|\boldsymbol{P}|\right\}$

Now, let $\boldsymbol{\Omega}=\boldsymbol{\chi}+\boldsymbol{\psi}$,

where,

$\left.\begin{array}{l}\boldsymbol{\Omega}_{c}=\boldsymbol{A}_{c}^{T} \boldsymbol{P}+\boldsymbol{P} \boldsymbol{A}_{c} \\ \boldsymbol{\Omega}_{r}=\boldsymbol{A}_{r}^{T}|\boldsymbol{P}|+|\boldsymbol{P}| . \boldsymbol{A}_{r}\end{array}\right\}$

Since, $\boldsymbol{A}^{T} \boldsymbol{P}+\boldsymbol{P} \boldsymbol{A}$ is always a symmetric matrix and $\left(\boldsymbol{A}^{T} \boldsymbol{P}+\right.$ $\boldsymbol{P A})=\boldsymbol{\Omega} \in\left[\boldsymbol{\Omega}_{c}-\boldsymbol{\Omega}_{r}, \boldsymbol{\Omega}_{c}+\boldsymbol{\Omega}_{r}\right]$

The condition is $\boldsymbol{A}^{T} \boldsymbol{P}+\boldsymbol{P} \boldsymbol{A}<0$, so the $\boldsymbol{\Omega}<0$

$\lambda_{\max }(\boldsymbol{\Omega}) \in\left[\lambda_{\max }\left(\boldsymbol{\Omega}_{c}\right)-\rho\left(\boldsymbol{\Omega}_{r}\right), \lambda_{\max }\left(\boldsymbol{\Omega}_{c}\right)+\rho\left(\boldsymbol{\Omega}_{r}\right)\right]$

or,

$$
\lambda_{\max }\left(\boldsymbol{\Omega}_{c}\right)+\rho\left(\boldsymbol{\Omega}_{r}\right)<0
$$

$\lambda_{\max }\left(\boldsymbol{\Omega}_{c}\right)<-\rho\left(\boldsymbol{\Omega}_{r}\right)$

which implies

$\lambda_{\max }\left(\boldsymbol{A}_{c}^{T} \boldsymbol{P}+\boldsymbol{P} \boldsymbol{A}_{c}\right)<-\rho\left(\boldsymbol{A}_{r}^{T}|\boldsymbol{P}|+|\boldsymbol{P}| . \boldsymbol{A}_{r}\right)$

where, $\lambda_{\text {max }}$ denotes the maximum Eigen value of the corresponding matrix and $\rho$ is defined as the spectral radius of the corresponding matrix. 


\section{SiMULATION AND RESULTS}

In this section, the proposed $H_{\infty}$ based FSFC has been simulated for a PWR for different scenarios. It can be noted that in this case the SFC is designed as a FSFC type $H_{\infty}$ controller. Practically, the measurement of all the system states of a PWR is not feasible due to limitations on sensors. Here, a Kalman filter is used to estimate all the states and it is demonstrated by a schematic diagram shown in Fig. 2.

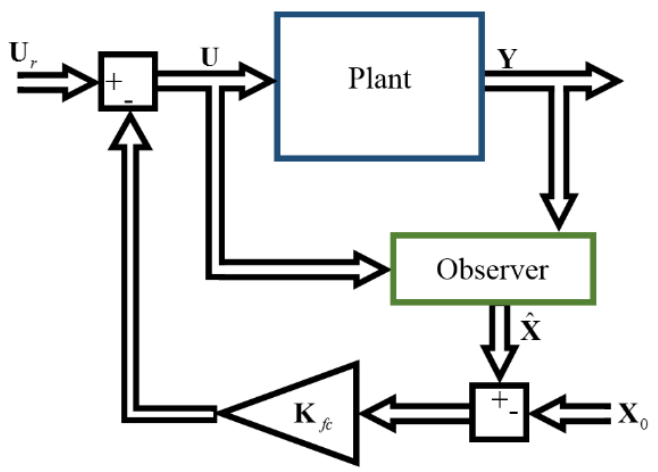

Fig. 2. Schematic diagram of controller with plant.

First, an interval system matrix for a PWR representing the $60 \%$ Full Power (FP)-100\% FP has been formulated as (12) with the help of the point kinetics and thermal hydraulic data [17]. Then, a $10 \%$ bounded parametric uncertainty has been considered on thermal hydraulic data to generate wide interval system matrix plant as (13). Next, a LMI of (26) as an interval plant has been solved by the YALMIP [20] and INTLAB [21] toolbox of MATLAB. Further, using INTLAB toolbox the stability criteria has been checked for the specified disturbance. If the stability is not guaranteed for the specified disturbance, then a reduction of the variance of disturbances might need to be considered.

It has been established by many researchers that the SFCs are not the tracking controller; SFCs are more acting as stabilizer rather than the tracking controller [21-22]. For this reason, it has been considered that the PWR is running on a $100 \% \mathrm{FP}$ and then at 20 second, an instantaneous change in the speed of control rod movement is considered which leads to a step disturbance in the reactivity. The control rod speed variation is shown in Fig. 3.

This disturbance directly affects the reactivity. Fig. 4 infers the change of the actuator output with and without controller in presence of disturbance. From Fig. 4, it is clear that without controller, a step disturbance in reactivity is shown while with the controller this step disturbance is rejected successfully.

Fig. 5 compares the actual power output with and without controller. As stated earlier that the PWR is an inherent stable system, with presence of disturbance it is still a stable system with a high offset error. The controller is performing well for rejecting the disturbances and for minimizing the offset error as well.

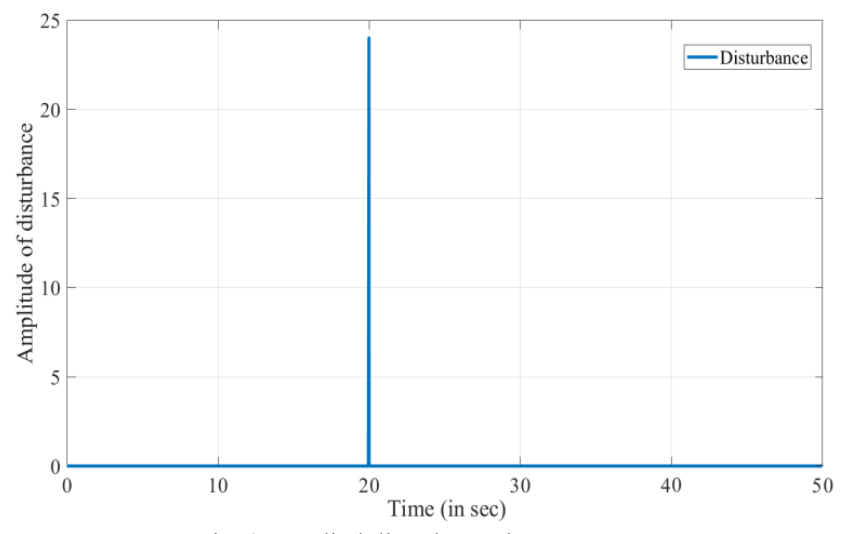

Fig. 3. Applied disturbance in actuator.

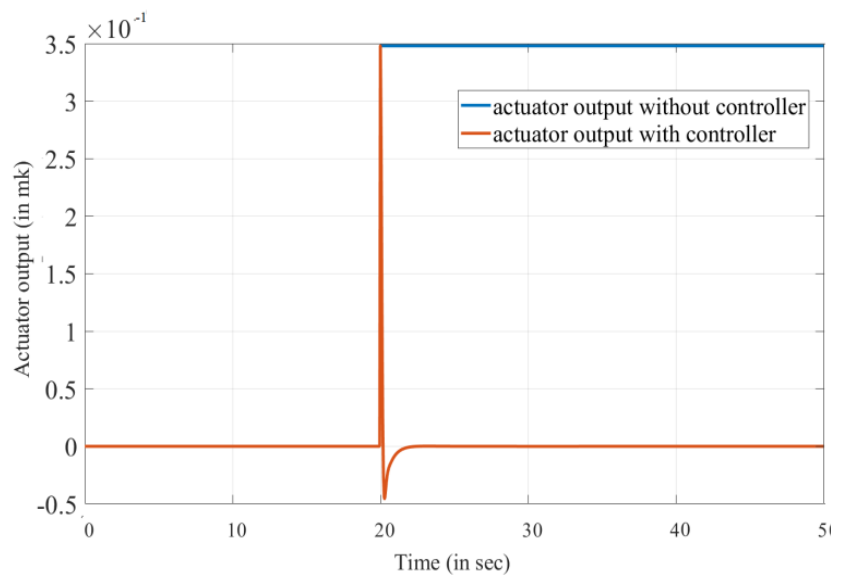

Fig. 4. Actuator output with and without controller.

As discussed earlier that FSFC are generally used as a stabilizing controller rather than a tracking controller; for tracking purpose a scaling factor $\boldsymbol{N}_{s}$ has been calculated to eliminate the steady state error described as in [23].

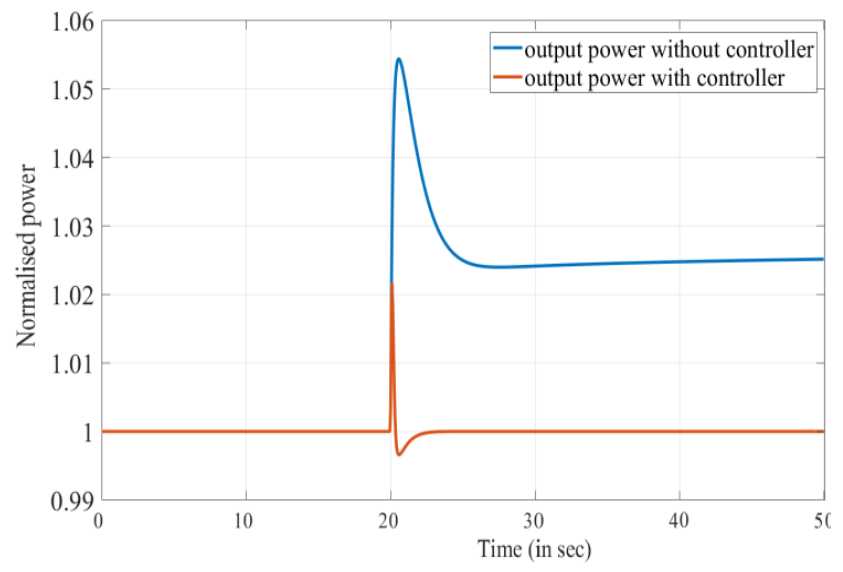

Fig. 5. Power output in presence of disturbance with and without controller. 


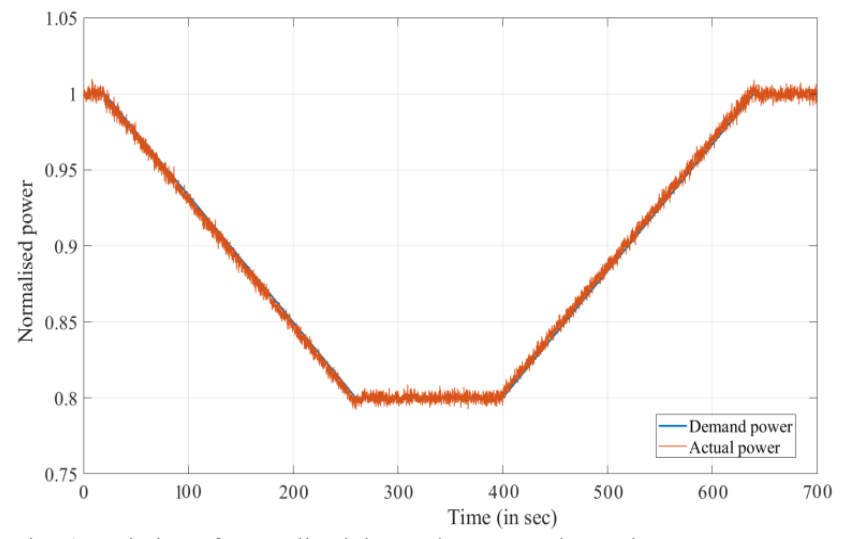

Fig. 6. Variation of normalized demand power and actual reactor power.

Initially it is assumed that the reactor is running at $100 \% \mathrm{FP}$. After 20 seconds, the demand power is reduced at a rate of $5 \% \mathrm{FP} / \mathrm{min}$ followed by a steady state power of $80 \% \mathrm{FP}$ for 140 seconds. Then, again the demand power is increased by $20 \% \mathrm{FP}$ at same rate. To check the robustness of the proposed controller $40 \mathrm{~dB}$ measurement noise in power is injected to the system. Fig. 6 depicts that the proposed controller can track the demand power in presence of measurement noise as well.

\section{CONCLUSION}

In this paper, a stability criterion is proposed to study the uncertainty handling capability of a FSFC approach. To establish an effectiveness of a robust FSFC, nonlinear PWR plant model has been used for simulation. The time domain performance of the proposed robust controller is found to be satisfactory for the disturbance. However, this robust controller is a suboptimal controller and could not guarantee the position of the closed loop poles.

\section{ACKNOWLEDGMENT}

The work presented in this paper has been supported by research grants from The Engineering and Physical Sciences Research Council (EPSRC) References: EP/R021961/1 and EP/R022062/1.

\section{REFERENCES}

[1] J. Wan, \& P. Wang, S. Wu, and F. Zhao, "Conventional Controller Design for the Reactor Power Control System of the Advanced Small Pressurized Water Reactor," Nuclear Technology, 2017.

[2] R. M., Edwards, K. Y. Lee, and M. A. Schultz, "State feedback assisted classical control: an incremental approach to control modernization of existing and future nuclear reactors and power plants," Nuclear technology 92, no. 2 (1990): 167-185.

[3] L. Liu, X.C. Luan, S. Rao, G.Y. Jin and Y.U. Tao, "Application of fuzzy robust control method in power control of nuclear reactors," Atomic Energy Sci. Technol. 47, 2013.
[4] A. P. Tiwari, G. D. Reddy, and B. Bandyopadhyay. "Design of periodic output feedback and fast output sampling based controllers for systems with slow and fast modes," Asian Journal of Control 14, no. 1 (2012): 271-277.

[5] R. Lamba, S. K. Singla, and S. Sondhi. "Fractional order PID controller for power control in perturbed pressurized heavy water reactor," Nuclear Engineering and Design 323 (2017): 84-94.

[6] R. M. Edwards, K. Y. Lee and A. Ray. "Robust optimal control of nuclear reactors and power plants," Nuclear Technology 98, no. 2 (1992): 137-148.

[7] K. J. Hwan, S. H. Park, and M. G. Na, "Design of a model predictive load-following controller by discrete optimization of control rod speed for PWRs," Annals of Nuclear Energy, vol. 71, pp. 343-351, 2014.

[8] H. Eliasi, M. B. Menhaj, and H. Davilu, "Robust nonlinear model predictive control for a PWR nuclear power plant," Progress in Nuclear Energy, vol. 54, no. 1, pp. 177-185, 2012.

[9] N. M. Gyun, D. W. Jung, S. H. Shin, J. W. Jang, K. B. Lee, and Y. J. Lee, "A model predictive controller for load-following operation of PWR reactors," IEEE Transactions on Nuclear Science, vol. 52, no. 4, pp. 10091020, 2005.

[10] Z. Dong, J. Feng and X. Huang. "Nonlinear observer-based feedback dissipation load-following control for nuclear reactors," IEEE Transactions on Nuclear Science, vol. 56, no. 1 pp. 272-285, 2009.

[11] K. Torabi, O. Safarzadeh and A. R. Moghaddam. "Robust control of the PWR core power using quantitative feedback theory," IEEE Transactions on Nuclear Science, vol. 58, no. 1, pp. 258-266, 2010.

[12] Z. Dong, "Adaptive proportional-differential power-level control for pressurized water reactors," IEEE Transactions on Nuclear Science, vol. 61, no. 2, pp. 912-920, 2014.

[13] D. Bose, S. Banerjee, M. Kumar, P. P. Marathe, S. Mukhopadhyay, and A. Gupta, "An Interval Approach to Nonlinear Controller Design for LoadFollowing Operation of a Small Modular Pressurized Water Reactor," IEEE Transaction on Nuclear Science, vol. 64, no. 9, pp. 2474-2488, Sept. 2017.

[14] D. Bose, S. Mukhopadhyay and A. Gupta, "An Optimal Nonlinear Dynamic Inversion Based Controller Design for Load-Following PWR," 2018 Second International Conference on Advances in Computing, Control and Communication Technology (IAC3T), Allahabad, India, pp. 75-80, 2018.

[15] K.J. Åström and B. Wittenmark, "Computer-Controlled Systems Theory and Design," 3rd ed. Englewood Cliffs, NJ: Prentice-Hall, 1997).

[16] X. Yan, P. Wang, J. Qing, S. Wu, and F. Zhao, "Robust power control design for a small pressurized water reactor using an $\mathrm{H}$ infinity mixed sensitivity method," Nuclear Engineering and Technology, 2020.

[17] S. E. Arda, and K. E. Holbert, "Nonlinear dynamic modeling and simulation of a passively cooled small modular reactor," Progress in Nuclear Energy 91, pp. 116-131, 2016

[18] X. Rong, X. Wang, and Y. Li. "H infinity state feedback control for the stabilization of the three Euler angles of helicopter based on LMI," In 2008 International Conference on Intelligent Computation Technology and Automation (ICICTA), vol. 1, pp. 375-379. IEEE, 2008.

[19] S. M. Rump, "Fast interval matrix multiplication," Numerical Algorithms 61, no. 1 (2012): 1-34

[20]YLMIPWiki[Online].Available:http://www.users.isy.liu.se/johanl/yalm ip/pmwiki.php?n=Main. WhatIsYALMIP

[21] S.M. Rump. INTLAB - INTerval LABoratory. In Tibor Csendes, editor, Developments in Reliable Computing, pages 77-104. Kluwer Academic Publishers, Dordrecht, 1999.

[22] H. Li, M. Y. Chow and Z. Sun, "EDA—based speed control of a networked DC motor system with time delays and packet losses," IEEE Trans. Ind. Electron. 56 (5) (2009) 1727-1735.

[23] H. Li, M. Y Chow and Z. Sun, "Speed control of a networked DC motor system with time delays and packet losses," in: 34th Annual Conference of IEEE Industrial Electronics, November 2008, pp. 2941-29. 